THE KURUME MEDICAL JOURNAL

Vol. 35, p. 201-205, 1988

\title{
Extragenital Malignant Mixed Müllerian Tumor: A Case Report
}

\author{
TAKASHI NISHIDA, TOSHIO OBUCHI, TOSHI SASAKI, NAOKI NAGASUE \\ AND MICHIAKI YAKUSHIJI* \\ Department of Obstetrics and Gynecology, National Kokura Hospital, ${ }^{*}$ Department of \\ Obstetrics and Gynecology, Kurume University School of Medicine, Kurume, 830 Japan
}

Received for publication October 13, 1988

\begin{abstract}
Summary: A case of an extragenital malignant mixed Müllerian tumor with heterologous elements is presented. The tumor originated from the pelvic peritoneum, as confirmed with a laparotomy and at autopsy. Combination chemotherapy with ifosfamide, adriamycin, cisplatin and etoposide resulted in a clinical remission, but the duration of the remission was limited. Histogenesis and therapeutic intractability are briefly discussed.
\end{abstract}

Key words: extragenital mixed Müllerian tumor - heterologous type - histogenesis - extended müllerian system - combination chemotherapy

\section{Introduction}

Malignant mixed Müllerian tumors (MMMT) are gynecological neoplasms which are not commonly observed. As the term indicates, the tumors usually arise in the tissues derived from the Müllerian system, e.g. the endometrium, endocervix, vagina and fallopian tubes. Since endometrioid type tumors can occur on the ovarian "coelomic" surface, MMMT may also be rarely observed in the ovary.

Extragenital MMMT is an extremely rare entity. According to a recent article by Chen and Wolk (1988), only seven cases, including their one patient, have been reported to have MMMT arising from extragenital sites, and three of the 7 tumors contained heterologous tissue components. The prognoses of the patients with extragenital MMMT were disappointing (Hasiuk et al, 1984; Chen and Wolk, 1988). Extensive intraperitoneal spreading at the time of the diagnostic laparotomy has prevented complete surgical excision, and no postoperative treatment can be recommended as truly effective therapy for MMMT (Lele et al. 1980).

Combination chemotherapy with ifosfamide, adriamycin and cisplatin (IAP) is currently used for patients with gynecological adenocarcinoma, including ovarian surface epithelial, endometrial and peritoneal malignancies, in the National Kokura Hospital. In recent clinical trials, a $100 \%$ response rate was obtained (Nishida and Nagasue, 1988; Nishida et al. 1988). From these encouraging results, IAP based combination chemotherapy was used to treat a patient with extragenital MMMT.

In this paper the fourth case of extragenital MMMT with heterologous elements is presented and the clinical trial of an IAP based regimen is described for this intractable disease.

Reprint requests to: Dr. Takashi Nishida: Department of Gynecology, National Kokura Hospital, Harugaoka 10-1, Kokura Minami-ku, Kitakyushu-Shi, Fukuoka-Ken, Japan 


\section{A Case Report}

A 51-year-old female (G7 P3) was admitted to the hospital on January 5,1988 , with a complaint of abdominal swelling associated with a slight dyspnea. There was no history of malignancies for her or her family. The laboratory data were not remarkable, except for a moderate leucocytosis. A chest $\mathrm{x}$-ray revealed a quantity of pleural effusion on the right side, and CT scanning and ultrasonography confirmed a large pelvic tumor, indistinguishable from the uterus and ovaries, that was associated with massive ascites. Cytological examinations of the ascites were positive, and led to an initial diagnosis of malignant ovarian neoplasm.

On January 8 , the patient underwent a diagnostic laparotomy. A large $(10 \times 9 \times$ $6-\mathrm{cm})$ tumor was found on the uterovesical peritoneum and there were diffuse intraabdominal tumor disseminations with bloody ascites $(3200 \mathrm{cc})$. Both ovaries and the uterus had normal appearances. A maximal debulking of the pelvic tumor, a total abdominal hysterectomy, a bilateral salpingo-oophorectomy and a partial omentectomy were performed.

Postoperatively, the patient received three courses of combination chemotherapy with ifosfamide $\left(1 \mathrm{~g} / \mathrm{m}^{2} \times 5\right)$, adriamycin $\left(50 \mathrm{mg} / \mathrm{m}^{2}\right)$ and cisplatin $\left(50 \mathrm{mg} / \mathrm{m}^{2}\right)$ plus etoposide $\left(100 \mathrm{mg} / \mathrm{m}^{2} \times 3\right)$ at four week intervals (IAP plus E). After two courses of IAP plus $E$ the patient went into a clinical remission, as indicated by the eradication of the pleural effusion and ascites, and an improved performance status. On June 14, following the 4 th course of IAP plus E, the disease flared up. Rapidly growing tumors were noted throughout the abdominal cavity, as well as ascitic fluid. Despite a change of the regimen and maximal supportive treatment, the patient died on August 26. An autopsy was performed.

\section{Pathologic Features}

A sectioned surface of the tumor which was resected during the laparotomy was solid, partly edematous, and hemorrhagic and necrotic in some focal areas. Histologically the tumor had features of MMMT, heterologous type (Fig. 1, 2, 3). The carcinomatous component was composed of columnar hyperchromatic cells arranged in irregular glands. In the sarcomatous area, oval or spindle-shaped cells proliferated occasionally forming a loose myxomatous meshwork. Rhabdomyoblasts with acidophilic cytoplasm and eccentric nuclei, proliferations of strap cells with or without demonstrable cross-striations were also observed in the malignant stroma containing chondroid differentiation. There were frequent mitotic figures. The uterus and ovaries were not affected by the tumor.

At autopsy the entire visceral peritoneum was thickened by confluent masses of extended tumors which encased the large and small intestines and were densely adhered to the abdominal wall. The lung and liver were also involved, while the gallblatdder was free from tumor tissue. Histologic examination confirmed the pathologic features observed in the antemortem laparotomy.

\section{Discussion}

The case presented is the fourth case of MMMT with heterologous elements arising from an extragenital site. The reported cases are briefly summarized in Table 1. The diagnosis of an extragenital origin for this tumor is based on the complete histologic examinations at laparotomy and autopsy. The Müllerian derivatives and ovaries must be excluded as histologic origins. All the reported cases, including the present case, suggest a peritoneal origin. Since the pelvic peritoneum has 


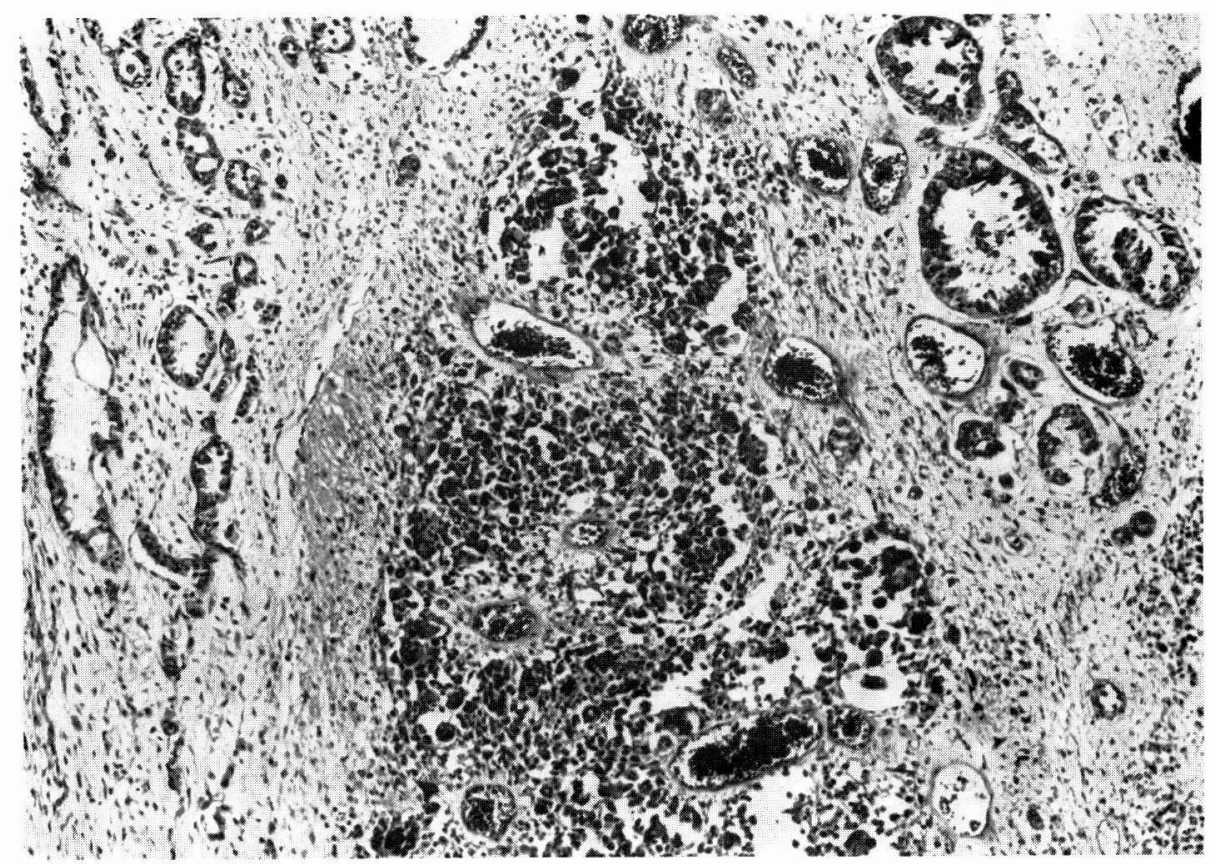

Fig. 1. Histology of a malignant mixed Müllerian tumor showing a mix ture of varied sarcomatous elements and an endometrioid type adenocar cinoma. (H. E. $\times 50)$

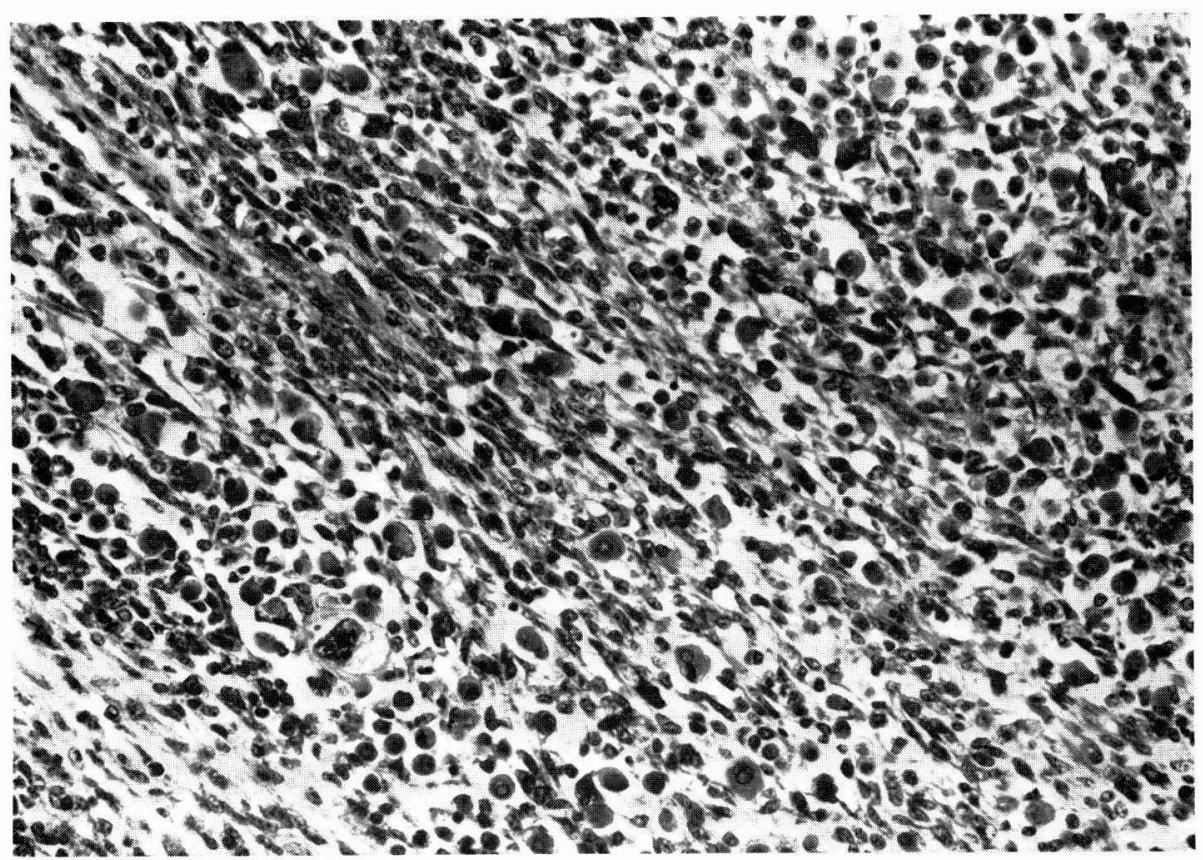

Fig. 2. Proliferated rhabdomyoblasts with acidophilic cytoplasm and eccentric nuclei mingled with a spindle cell sarcomatous component. $($ H. E. $\times 100)$ 




Fig. 3. Malignant stromal cells with occasional chondroid differentiation (left side). (H.E. $\times 100)$

TABLE 1

Reported cases of extragenital MMMT

\begin{tabular}{llllrrr}
\multicolumn{1}{c}{ author } & year & age & \multicolumn{2}{c}{ origin } & MMMT type & follow up \\
\hline 1. Ober and Balck & 1955 & 74 & rectovaginal peritoneum & homo & DOD & $5 M$ \\
2. De La Pava, et al. & 1963 & $45^{*}$ & pelvic peritoneum & homo & DOD & $4 \mathrm{M}$ \\
3. Ferrie and Ross & 1967 & 47 & posterior peritoneum & homo & ? \\
4. Weisz-Carrington, et al. & 1977 & 77 & cecal peritoneum & hetero & DOD & $1 W$ \\
5. Hasiuk, et al. & 1984 & 77 & posterior peritoneum & hetero & DOD & $1 \mathrm{M}$ \\
6. Nguyen and Berendt & 1986 & 58 & omentum & hetero & homo & DOD $11 \mathrm{M}$ \\
7. Chen and Wolk & 1988 & $57^{* *}$ & pelvis & hetero & DOD $8 M$ \\
8. Present case & 1988 & 51 & pelvic peritoneum & &
\end{tabular}

homo : homologous type hetero: heterologous type DOD : died of disease
* Patient received prior radiation therapy for endometriosis.

** Patient received previous cytotoxic chemotherapy for ovarian cancer. the capability to differentiate in the Müllerian directions, as "the secondary Müllerian system" (Lauchlan, 1972), the origin of this neoplasm can be explained by the heterotopic Müllerian potentiality of the pelvic peritoneum. Although some authors have suggested that previous cytotoxic treatment is a tumorigenic factor (Table 1: case 2,7 ), no patients in the other reports had such a history.

The postoperative survival in reported cases with extragenital MMMT was short 
(heterologous type, 2. 5 weeks on the average). There is a general agreement that MMMT is unresponsive to therapy. The only effective treatment for patients with MMMT is probably surgical resection of the localized malignancies (Krupp et al. 1961; Lele et al. 1980). Our patient treated with IAP plus E survived for eight months, postoperatively. The objective response and an improved performance status permitted her to leave the hospital transiently, but the duration was limited.

IAP is a combination regimen modified from CAP (cyclophosphamide, adriamycin and cisplatin), which is the most widely accepted regimen for patients with ovarian cancer. An IAP based combination, IAP plus etoposide, was used for the patient with Müllerian non-epithelial malignancy in this report. When considering the therapeutic resistance of MMMT to cytotoxic therapy, the results presented here are promising. Further investigation will confirm the true efficacy of IAP plus $E$ for patients with gynecological nonepithelial malignancies.

\section{References}

Chen, K.T. and Wolk, R.W. (1988), Extragenital mixed Müllerian tumor. Gynecol. Oncol. 30, 422-426.

De La Pava, S., Nigogosyan, G. and Pickren, J. W. (1963). Sarcomatous transformation of "true" endometriosis. N. Y. J. Med. 63, 25482553.

FERRIE, R. K. and Ross, R.C. (1967). Reteroperi- toneal müllerian carcinosarcoma. Canad. Med. Ass. J. 97, 1290-1292.

Hasiuk, A.S., Petersen, R.O., Hanjani, P. and Griffin, T.D. (1984), Extragenital malignant mixed müllerian tumor. Case report and review of the literature. Am. J. Clin. Pathol. 81, 102-105.

Krupp, P. J., Sternberg, W. H., Clark, W. H., ST. Romain, M. J. and Smith, R. C. (1961), Malignant mixed müllerian neoplasms (mixed mesodermal tumors). Am. J. Obstet. Gynecol. 81, 959-969.

Lauchlan, S.C. (1972), The secondary müllerian system. Obstet. Gynecol. Surv. 27, 133-146.

Lele, S. B., Piver, M.S. and Barlow, J. (1980), Chemotherapy in management, of mixed mesodermal tumors of the ovary. Gynecol. Oncol. 10, 298-302.

Nguyen, G. and Berendt, R.C. (1986), Aspiration biopsy cytology of metastatic endometrial stromal sarcoma and extragenital mixed mesodermal tumor. Diag. Cytopathol. 2, 256-260.

Nishida, T. and Nagasue, N. (1988). Treatment of Gynecological adenocarcinomas with a combination of ifosfamide, adriamycin and cisplatin. Acta Obstet. Gynecol. Jpn. 40, 635639.

Nishida, T., Nagasue, N., Kishi, N., Imaishi, K., Tateno, N., Matsumura, T. and Yakushiji, M. (1988), Preliminary study of a new combination therapy with ifosfamide, adriamycin and cisplatin for uterine endometrial cancer. Acta Obstet. Gynecol. Jpn. 40, 1883-1888.

OBer, W. B. and Black, M. B. (1955), Neoplasms of the subcoelomic mesenchyme. Arch. Pathol. Lob. Med. 59, 698-705.

Weisz-Carrington, P., Biglow, B. and Schinella, R. A. (1977). Mixed heterologous tumor of Muillerian type arising in the recal peritoneum, report of a case. Dis. Colon Rectum. 20, 329333. 